\title{
Four-dimensional velocity encoded MRI improves blood flow quantification in patients with semilunar valve stenosis
}

\author{
Sarah Nordmeyer, Eugenie Riesenkampff, Daniel Messroghli, Felix Berger, Titus Kuehne \\ From 15th Annual SCMR Scientific Sessions \\ Orlando, FL, USA. 2-5 February 2012
}

\section{Background}

Stenoses might cause complex flow patterns, which are sometimes difficult to assess quantitatively with standard two-dimensional (2D) VEC MRI. We sought to evaluate the use of four-dimensional (4D) velocity encoded magnetic resonance imaging (VEC MRI) for blood flow quantification in patients with semilunar valve stenosis.

\section{Methods}

Peak velocities (Vmax) and stroke volumes (SV) were quantified by $2 \mathrm{D}$ and $4 \mathrm{D}$ VEC MRI in volunteers $(\mathrm{n}=7)$ and patients with semilunar valve stenosis $(n=18)$. Measurements were performed immediately above the aortic or pulmonary valve with both techniques (=level 1) and, additionally, at further predefined planes in the ascending aorta and in the pulmonary trunk within the $4 \mathrm{D}$ dataset. 4D VEC MRI streamline analysis identified "individualized" planes of highest flow velocity (4Dmaxtargeted) for further measurements. In patients, Vmax was also measured by Doppler-echocardiography.

\section{Results}

In patients, 4D VEC MRI $(2.7 \mathrm{~m} / \mathrm{s})$ showed comparable Vmax to Doppler-echocardiography $(2.8 \mathrm{~m} / \mathrm{s})$ and significantly higher Vmax than 2D VEC MRI $(2.4 \mathrm{~m} / \mathrm{s})$ $(\mathrm{p}<0.03)$ at level 1. 4Dmax-targeted revealed highest Vmax values $(3.1 \mathrm{~m} / \mathrm{s})$. Correlations of MR-derived peak velocities with Doppler-echocardiography were $r=0.62$ for $2 \mathrm{D}, \mathrm{r}=0.67$ for $4 \mathrm{D}$ at level 1 and $\mathrm{r}=0.80$ for $4 \mathrm{Dmax}-$ targeted. 4D showed higher agreement with Dopplerechocardiography than did 2D VEC MRI at level 1 $(\mathrm{p}=0.039)$.

\footnotetext{
Department of Congenital Heart Disease/Pediatric Cardiology, Deutsches Herzzentrum Berlin, Berlin, Germany
}

(c) 2012 Nordmeyer et al; licensee BioMed Central Ltd. This is an open access article distributed under the terms of the Creative Commons Attribution License (http://creativecommons.org/licenses/by/2.0), which permits unrestricted use, distribution, and reproduction in any medium, provided the original work is properly cited.

\section{and take full advantage of:}

- Convenient online submission

- Thorough peer review

- No space constraints or color figure charges

- Immediate publication on acceptance

- Inclusion in PubMed, CAS, Scopus and Google Scholar

- Research which is freely available for redistribution 
Table 1 Comparison between 2D (level 1) and 4D (level 1 and 4D max-targeted) derived peak velocity (+Dopplerechocardiography) in the ascending aorta in patients with aortic valve stenosis and in the pulmonary trunk in patients with pulmonary valve stenosis

\begin{tabular}{ccccc}
\hline Patients & $V \max (\mathrm{m} / \mathrm{s}) \mathrm{ECHO}$ & $V \max (\mathrm{m} / \mathrm{s})$ 2D Level 1 & Vmax $(\mathrm{m} / \mathrm{s})$ 4D Level 1 & Vmax (m/s) 4D max-targeted \\
\hline Mean & 2.78 & 2.40 & 2.74 & 3.12 \\
SD & 0.68 & 0.54 & 0.64 & 0.63 \\
p-value* & 0.009 & - & 0.025 & $<0.001$ \\
p-value** & - & $<0.01$ & $>0.05$ & $<0.05$ \\
\hline
\end{tabular}

Vmax $=$ peak velocity, $2 \mathrm{D}=$ two-dimensional, $4 \mathrm{D}=$ four-dimensional, $\mathrm{SD}=$ standard deviation, Echo $=$ Doppler-echocardiography, ${ }^{*}=$ compared to $2 \mathrm{D},{ }^{* *}=$ compared to Echo
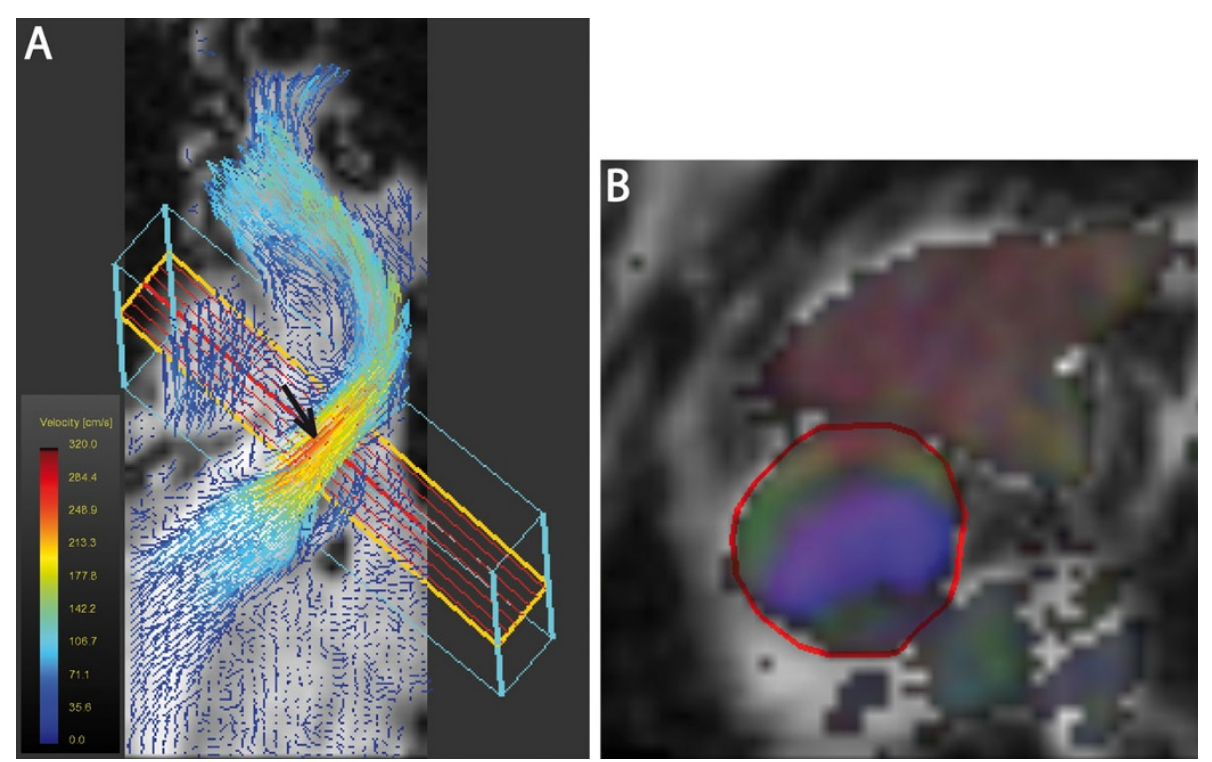

Figure 1 Assessment of peak velocity using flow visualization by 4D VEC MRI Figure: Velocity information in the left ventricular outflow tract of a patient with bicuspid aortic valve. A) Coronal axis of the left ventricular outflow tract, aortic valve and ascending aorta with in-plane visualization of velocity information. Dark red (black arrow) visualizes site of peak velocity. B) 4Dmax-targeted plane for blood flow quantification with colour coded flow direction. 